\title{
Avaliação de habilidades clínicas e feedback na residência médica em Pediatria
}

\author{
Clinical skills assessment and feedback in pediatric residency
}

\author{
Bruna de Sá Duarte Auto' (1) | brunaduarte100@gmail.com \\ Maria Viviane Lisboa de Vasconcelos' ${ }^{1}$ (D) camposdelisboa@gmail.com

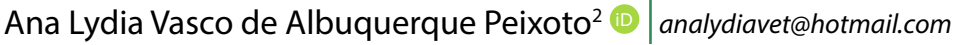

\section{RESUMO}

Introdução: A residência médica é a especialização por excelência na formação do médico, e cabe ao programa assegurar que o residente egresso atinja o nível almejado de competência. Um sistema avaliativo bem elaborado e com feedback é ferramenta efetiva para aprimorar o desempenho do futuro especialista e garantir a qualificação dele.

Objetivo: Este estudo teve como objetivo analisar o sistema de avaliação do médico residente em pediatria de um hospital universitário, com o intuito de promover a formação docente em métodos avaliativos.

Método:Trata-se de uma pesquisa-ação educacional (pesquisa-ensino) realizada com docentes e preceptores da residência médica em pediatria de um hospital universitário. As etapas consistiram em: 1. aplicação de questionário sobre o perfil dos participantes e os métodos avaliativos utilizados com os residentes; 2 . intervenção com a realização de um workshop sobre avaliação de habilidades clínicas e feedback; 3 . avaliação imediata, após o workshop, com aplicação de outro questionário, elaborado com base no nível 1 do método Kirkpatrick. Utilizaram-se a análise estatística simples, para os dados objetivos, e a análise de conteúdo, segundo recomendações de Malheiros e Bardin, para a parte qualitativa.

Resultado: Dos 21 participantes, dez (48\%) informaram que não tinham capacitação formal em avaliação e que utilizavam métodos avaliativos mais tradicionais. Quanto aos métodos, 81\% (17/21) dos participantes informaram que utilizavam mais de um, com finalidade somativa, para obter uma avaliação mais abrangente e fidedigna. No entanto, nenhum utilizava uma avaliação sistematizada de habilidades clínicas com fornecimento de feedback. Após o workshop com enfoque em avaliação de desempenho em ambiente simulado, em que se adotou o Objective Structured Clinical Examination (OSCE), os participantes utilizaram, com os internos, o método avaliativo do aprendizado na sua prática cotidiana, e, dessa forma, o treinamento atingiu o nível 3 de Kirkpatrick.

Conclusão: A pesquisa-ação propiciou identificar limitações no sistema de avaliação e feedback do médico residente em pediatria. A metodologia utilizada revelou um efeito agregador e contribuiu para desenvolver o sentido colaborativo e integrativo no grupo. No entanto, não foi suficiente para interferir positivamente, em curto prazo, na avaliação da residência médica em pediatria.

Palavras-chave: Residência Médica; Feedback Formativo; Capacitação de Professores; Avaliação de Desempenho.

\section{ABSTRACT}

Introduction: A medical residency is the specialization par excellence in medical training and the program is responsible for ensuring that the recently graduated doctor reaches the established level of competence. A well-prepared assessment system with feedback is an effective tool to enhance the performance of future specialists and guarantee their qualification.

Objective: To analyze the assessment system for pediatric residents at a university hospital, aiming to promote teaching training in assessment methods.

Methodology: educational action research (research-teaching), conducted with teachers and preceptors in the pediatric residency of a university hospital. The stages consisted of: a) the application of a questionnaire on participant profile and assessment methods used to evaluate the medical residents; $b$ ) intervention (workshop) on the assessment of clinical skills and feedback; c) immediate assessment after the workshop, by applying another questionnaire based on level 1 of the Kirkpatrick model. Simple statistical analysis was used for the objective data and the content analysis, according to recommendations by Malheiros (2011) and Bardin (2013), for the qualitative part.

Result: Ten (48\%) of the 21 participants declared not being formally trained in assessment and that they applied the more traditional methods learned from personal experience. Regarding the assessment methods, 81\% (17/21) of the participants used more than one, aiming to obtain a more encompassing and reliable assessment. However, none of the teachers/preceptors used a systematized assessment of the clinical skills or provided feedback to the medical residents. After the workshop, with a focus on assessing performance in a simulated 'Objective Structured Clinical Examination' (OSCE) environment, all the participants were favorable to apply the OSCE in their teaching-learning practice with residents and undergraduate students, reaching Kirkpatrick level 3.

Conclusion: The action research helped identify limitations in the assessment and feedback system of the pediatric resident physician. The methodology used revealed an aggregating effect and contributed to the development of the collaborative and integrative sense in the group. However, it was not enough to positively interfere with the pediatric medical residency assessment in the short term.

Keywords: Medical Residency; Formative Feedback; Teacher Training; Performance Assessment.

${ }^{1}$ Universidade Federal de Alagoas, Maceió, Alagoas, Brasil.

2 Universidade Estadual de Ciências da Saúde de Alagoas, Maceió, Alagoas, Brasil.

Editora-chefe: Daniela Chiesa

Editora associada: Daniela Chiesa

Recebido em 27/12/19; Aceito em 19/04/21. Avaliado pelo processo de double blind review. 


\section{INTRODUÇÃO}

A residência médica é a especialização por excelência na formação do médico, e cabe ao programa assegurar que o egresso atinja o nível almejado de competência'. Por ser um treinamento em serviço, avaliar, nesse contexto, vai além da análise cognitiva, sendo um desafio diário para o docente e o preceptor.

Um processo avaliativo eficaz requer, além de formação docente, um sistema que combine diversas formas de avaliação, de modo a atingir todos os elementos do aprendizado (conhecimentos, habilidades e atitudes), além de assegurar a validade e a fidedignidade dos métodos utilizados².

Diante da complexidade da atuação do docente na educação médica, a elaboração e a implementação de programas de desenvolvimento docente, atividades que buscam aprimorar os saberes e habilidades dos profissionais de saúde como professores, devem ser consideradas um processo permanente ${ }^{3,4}$. Porém, segundo Steinert ${ }^{4}$, apesar do crescente interesse pelo tema nos últimos anos, poucos trabalhos focaram, especificamente, o desenvolvimento docente para avaliação do aluno.

O cenário desta pesquisa - nossa prática cotidiana como docentes e preceptores na área de pediatria - é o Hospital Universitário Professor Alberto Antunes (Hupaa), da Universidade Federal de Alagoas (Ufal), que conta com um programa de residência médica (PRM) em pediatria instituído há 30 anos e porta todas as credenciais para mantê-lo vigente até os dias atuais. O programa tem duração de três anos, e são disponibilizadas, anualmente, cinco vagas. A taxa de ocupação da residência médica em pediatria, de 2015 aos dias atuais, foi de $93,3 \%$ das vagas (28/30), com formação de $100 \%$ dos residentes $(18 / 18)^{5}$.

No entanto, o processo de avaliação dos residentes do programa ainda apresenta um caráter tradicional e somativo, apesar de a Comissão Nacional de Residência Médica (CNRM) definir diretrizes para a avaliação do médico residente desde a resolução CNRM n 05, de 12 de novembro de $1979^{6}$ (revogada), diretrizes reiteradas na resolução CNRM n 02, de 17 de maio de 2006, considerando-se o disposto no artigo 137:

Art. 13. Na avaliação periódica do médico residente serão utilizadas as modalidades de prova escrita, oral, prática ou de desempenho por escala de atitudes, que incluam atributos tais como: comportamento ético, relacionamento com a equipe de saúde e com o paciente, interesse pelas atividades e outros a critério da COREME da instituição. $\S 1^{\circ}$. A freqüência mínima das avaliações será trimestral. $§ 2^{\circ}$. A critério da instituição poderá ser exigida monografia e/ou apresentação ou publicação de artigo científico ao final do treinamento. $\S 3^{\circ}$ Os critérios e os resultados de cada avaliação deverão ser do conhecimento do médico residente.
Com relação à avaliação, um modelo conceitual proposto por Miller, há várias décadas, conhecido como pirâmide de Miller, demonstrou aos docentes que, no desenvolvimento profissional, a avaliação não pode ficar restrita aos conhecimentos teóricos, pois é necessário que o estudante saiba aplicar esses conhecimentos, executá-los, de maneira prática, em ambientes simulados e, finalmente, aplicálos na vida real ${ }^{8}$.

A pirâmide de Miller alinha os seus estratos com os objetivos educacionais e os métodos avaliativos direcionados aos tipos de habilidade e competência cujo domínio se quer conhecer, ascendendo do conhecimento teórico contido na base - "saber"e "saber como" - para o "mostrar como" e o "fazer". O ápice da pirâmide corresponde à avaliação do profissional no seu ambiente de trabalho ${ }^{9}$.

Com base nessas dimensões e no grau de aprendizagem do médico residente, as observações realizadas por docentes e preceptores devem ser direcionadas, além do cognitivo, para as avaliações de desempenho, considerando as habilidades clínicas e psicomotoras, a interação com o paciente, o manejo da informação, a capacidade de julgamento, de síntese e de decisão, bem como a preservação de atitudes éticas ${ }^{1}$.

A maioria dos métodos avaliativos de habilidades clínicas tem, como princípio básico, a observação direta do desempenho do residente em tarefas clínicas, em ambiente real ou simulado. Nesse sentido, deve permitir a realização do feedback, preferencialmente imediato (formativo), que consiste em descrever e discutir com o residente a sua performance em determinada atividade ${ }^{10}$.

Um sistema avaliativo bem elaborado e periódico, com feedback contínuo, é ferramenta efetiva para aprimorar o desempenho do futuro especialista e garantir a sua qualificação, meta de importância indiscutível no processo de formação Para tanto, a avaliação do residente necessita de sistematização e de institucionalização quanto ao modo de avaliar, além de formação docente para esse importante aspecto do processo de ensino-aprendizagem.

Esta pesquisa propôs responder ao seguinte questionamento:

- Como vêm sendo avaliados os residentes quanto às competências adquiridas no programa de residência médica em pediatria do Hospital Universitário Professor Alberto Antunes?

Dessa maneira, o objetivo foi analisar o sistema de avaliação do médico residente em pediatria de um hospital universitário, com o intuito de promover formação docente em métodos avaliativos. 


\section{PERCURSO METODOLÓGICO}

Para identificar lacunas na prática pedagógica e provocar mudanças nos hábitos educativos, idealizou-se uma pesquisaação educacional, devido às suas potencialidades como práxis investigativa no processo de avaliação do residente, por meio de uma amostra intencional com docentes e preceptores do referido programa de residência em pediatria.

No campo educacional, a pesquisa-ação (pesquisaensino) consiste na investigação sobre a própria prática e implica a tomada de consciência por parte dos participantes, permitindo envolvê-los em todas as fases do percurso metodológico ${ }^{11,12}$.

Sua utilização no campo educacional permite ao docente pesquisador identificar um problema na sua atividade pedagógica e, por meio da pesquisa, criar condições para transformá-la, a fim de favorecer o crescimento pessoal e profissional dos pesquisadores e dos participantes envolvidos ${ }^{11,12}$.

A pesquisa-ação segue um ciclo em que se aprimora a prática no movimento entre agir no campo do problema e investigar a respeito dela. O ciclo inclui a identificação do problema e a produção de dados sobre os efeitos de uma mudança na prática durante a intervenção, antes e depois da sua implementação, utilizando métodos pré e pós para monitorar os efeitos decorrentes da mudança ${ }^{13}$ (Figura 1).

Segundo Malheiros ${ }^{14}$, essa metodologia é muito útil no campo educacional, porque permite estudos quanto a alterações de currículo, modelos de ensino-aprendizagem, métodos de avaliação, entre outros aspectos.

No desenvolvimento da pesquisa, foram utilizados diferentes procedimentos para a coleta de dados, dividida

Figura 1. Diagrama representando o desenvolvimento da pesquisa-ação.

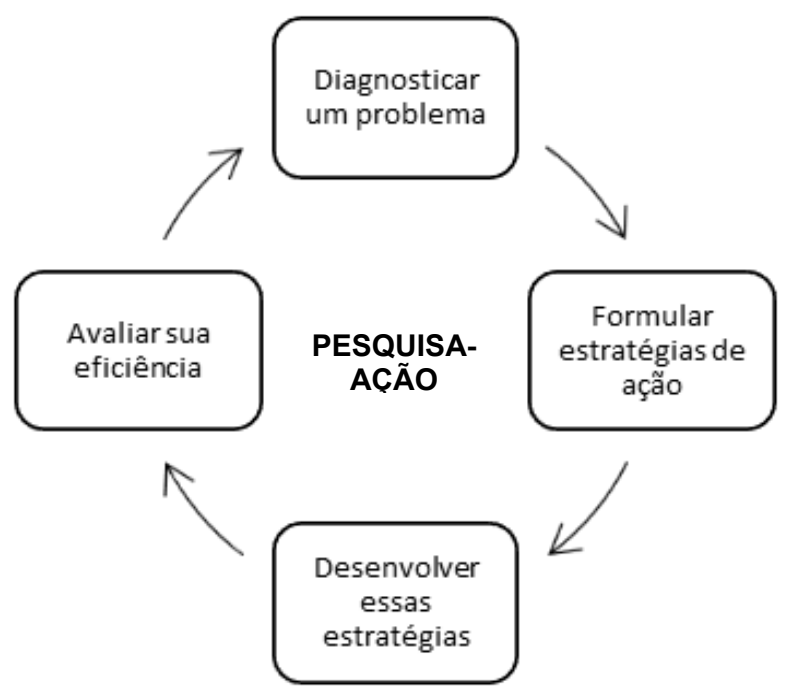

Fonte: Adaptada de Tripp ${ }^{13}$. em três etapas, observando a metodologia da pesquisaação e atendendo a ela, no intuito de entender a realidade da avaliação do médico residente e de propor estratégias de ação para sua melhoria.

Na etapa 1 do ciclo da pesquisa-ação, convidaram-se todos os docentes e preceptores que atuavam na residência médica em pediatria do Hupaa a participar da pesquisa, perfazendo o total de 22 envolvidos, sendo 16 docentes e seis preceptores, no período de janeiro de 2018 a abril de 2019. Dos 22 participantes, 16 eram vinculados diretamente ao Hupaa, e seis, a outros serviços de saúde (Hospital Geral do Estado Dr. Oswaldo Brandão Vilela e Hospital Escola Dr. Hélvio Auto), onde os residentes de pediatria do Hupaa atuam nos setores de urgência e emergência e doenças infectocontagiosas, respectivamente. Nessa fase, foi excluído da pesquisa um docente pesquisador, e os demais aceitaram participar na discussão e na resposta ao questionário.

Para identificar as deficiências no processo de avaliação dos residentes, aplicou-se o primeiro questionário semiestruturado, composto de questões referentes aos dados sociodemográficos dos participantes, aos dados específicos sobre capacitação docente em avaliação, aos métodos avaliativos (testes cognitivos ou avaliação de habilidades clínicas) que utilizam com os médicos residentes, ao conhecimento factual de métodos de avaliação de desempenho em ambientes reais e simulados, à visão dos docentes e preceptores quanto ao processo avaliativo, bem como à intenção de participarem de um workshop de desenvolvimento docente sobre um método avaliativo.

As respostas dos participantes às questões abertas foram organizadas com base nas ideias que emergiram das questões norteadoras, quando se realizou a pré-análise por meio da leitura mais aprofundada, observando-se a emergência de categorias não criadas previamente. Criou-se uma matriz, e todos os depoimentos foram transcritos ipsis litteris. Os participantes foram codificados por letras e números, seguindo a ordem de análise dos questionários - docente (D) e preceptor (P).

Criaram-se matrizes que armazenaram as ideias explícitas ou implícitas, a formação das categorias e as unidades de registro que relacionam os depoimentos com o tema para explicar, no texto, como se chegou ao resultado. Os focos e as unidades de registro foram interpretados, e procedeu-se à elaboração da síntese para cada foco ${ }^{14}$.

Utilizou-se a análise estatística simples, para os dados objetivos, e a análise de conteúdo, segundo recomendações de Malheiros ${ }^{14}$ e Bardin ${ }^{15}$, para a parte qualitativa.

A etapa 2, ação decorrente da busca de uma solução para o problema diagnosticado, levou ao planejamento de uma intervenção pedagógica. Segundo Malheiros ${ }^{14}$, 
nesse modelo de pesquisa-ação, uma intervenção é feita numa determinada realidade para, posteriormente, os seus resultados serem avaliados.

Essa etapa consistiu no planejamento da fase de intervenção, após a análise das respostas ao questionário. Prosseguiu-se com a construção do workshop de capacitação em avaliação de habilidades clínicas, para os docentes e preceptores, com enfoque no método avaliativo escolhido pelos participantes.

Com o objetivo de manter a estratégia integrativa, as datas para o treinamento foram consensuadas com todos os envolvidos (docentes, preceptores, atores e monitores da pediatria). Todo o processo do workshop foi registrado por meio de fotos e filmagens, com consentimento dos participantes.

A etapa 3 desse ciclo, três semanas após o workshop, caracterizou-se pela avaliação da intervenção pedagógica por meio de um questionário semiestruturado, enviado por correio eletrônico, com base no modelo de Kirkpatrick, que consiste em um modelo de avaliação consagrado internacionalmente e tem a finalidade de avaliar ações educativas voltadas para profissionais. É composto de quatro níveis de avaliação do treinamento: 1. reação, 2. aprendizado, 3. comportamento (transferência) e 4. resultados ${ }^{16}$.

O questionário foi elaborado com base no nível 1 do modelo de Kirkpatrick. Nesse nível, avalia-se a reação dos participantes ao treinamento em si, bem como as suas reações à experiência de aprendizado. Questionaram-se elementos como o conteúdo programático, as expectativas atingidas, a avaliação da infraestrutura e da logística (instalações e equipamentos), a duração e a organização do treinamento, a qualidade e o conteúdo do material didático, a estrutura dos cenários, assim como a avaliação dos palestrantes (didática, comunicação, interação e conhecimento) e a metodologia utilizada. Além disso, havia perguntas abertas sobre a motivação para participar da oficina e a disposição para utilizar o Objective Structured Clinical Examination (OSCE) na prática docente com os médicos residentes.

Com base no ciclo da pesquisa-ação, descrevem-se, no fluxograma a seguir, os caminhos percorridos, com as estratégias de investigação e as técnicas de análise dos dados construídas (Figura 2).

O projeto foi aprovado pelo Comitê de Ética em Pesquisa (CEP) da Ufal, sob o Parecer no 2.304 .092 Certificado de Apresentação para Apreciação Ética (CAAE) no 74854717.0.0000.5013, não havendo conflitos de interesse.

\section{RESULTADOS E DISCUSSÃO}

\section{Etapa 1 - Diagnóstico situacional}

Participaram dessa etapa, relativa ao diagnóstico do problema, todos os professores e preceptores que atuavam na residência médica em pediatria e que preencheram os critérios de inclusão, totalizando 21 , sendo 15 docentes e seis preceptores. Desses, 76\% (16/21) são do sexo feminino, com idades variando de 29 a 62 anos (média de 46,5 anos); tempo de formação, de sete a 38 anos (média de 22,3 anos); e tempo de docência, de um a 37 anos (média de 13,6 anos). Quanto à titulação, 23,8\% (5/21) possuem doutorado; e 33,3\%, mestrado (7/21).

Dos 21 participantes, dez (48\%) informaram que não tinham capacitação formal em avaliação e que utilizavam métodos avaliativos tradicionais, por experiência própria no ambiente de serviço. Nesse percentual, estão inclusos todos os preceptores, o que recai sobre o fato de não haver uma exigência de formação pedagógica para o exercício da preceptoria ${ }^{17}$, apesar de ser papel do preceptor ensinar, acompanhar na prática diária e avaliar os médicos em formação. Os preceptores, além do bom conhecimento específico na área de atuação, precisam de atividades de desenvolvimento docente e de apoio institucional permanente que os auxiliem no aprimoramento de suas habilidades de ensino ${ }^{18}$.

Quanto aos métodos avaliativos, 81\% (17/21) dos participantes informaram utilizar mais de um, com finalidade somativa, para obter uma avaliação mais abrangente e

Figura 2. Fluxograma da pesquisa.
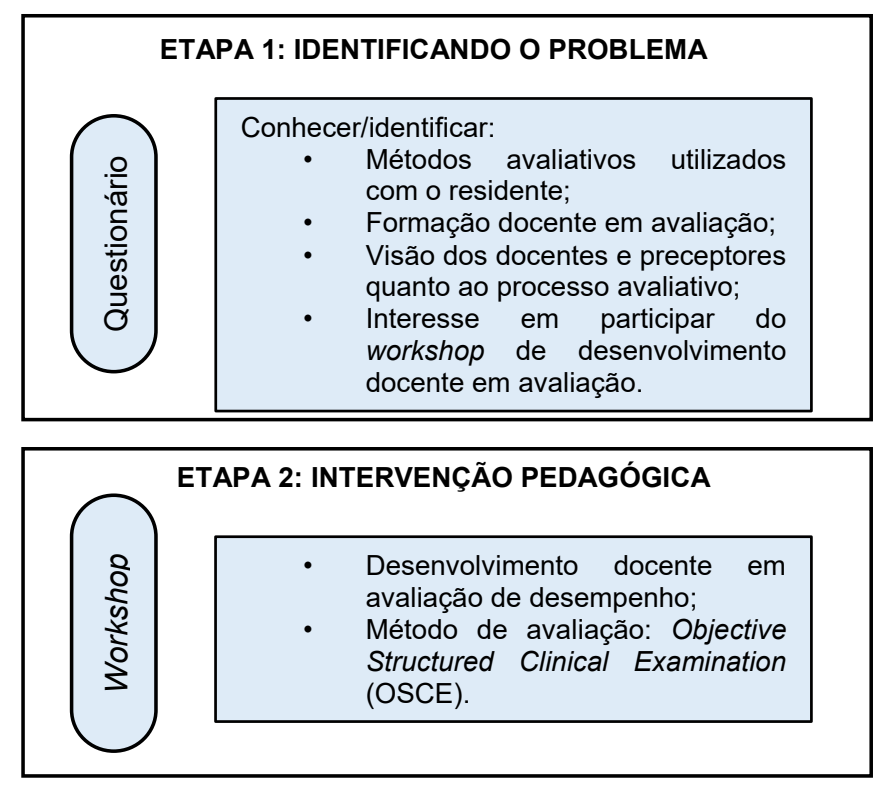

ETAPA 3: AVALIANDO A EFICÁCIA DA INTERVENÇÃO

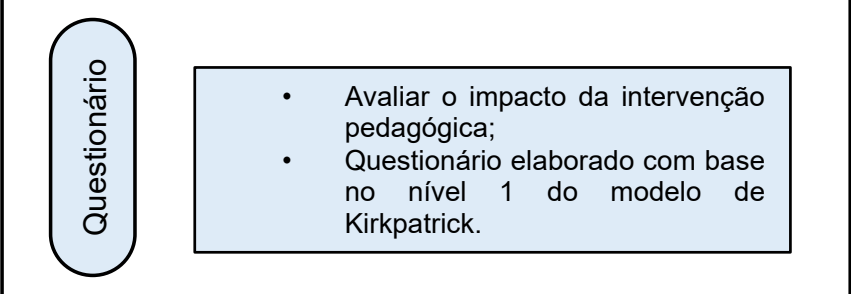

* A análise de dados e conteúdo foi realizada segundo as recomendações de Malheiros ${ }^{14}$ e Bardin $^{15}$. 
fidedigna. Segundo Norcini et al. ${ }^{19}$, a estrutura para uma boa avaliação consiste na combinação organizada de métodos, com o intuito de compor um sistema de avaliação, contudo nenhum dos docentes/preceptores utiliza uma avaliação sistematizada de habilidades clínicas, psicomotoras ou afetivas do médico residente, nem fornece feedback. Dessa forma, apesar da diversidade de métodos avaliativos, não há garantia de ausência de fragilidades ${ }^{20}$.

Nos depoimentos dos docentes e preceptores, pode-se evidenciar uma preocupação coletiva com essa inadequação nos métodos avaliativos:

Ótima pesquisa. Sempre me incomodou a avaliação sem recursos. Acredito que há riscos de injustiça (D2).

Reconheço a pobreza nos métodos tradicionais de avaliação. Talvez por acomodação e resistência às mudanças ainda não conseguimos sair desta zona de conforto. Aberto a novas experiências (D6).

Esses depoimentos corroboram os resultados obtidos por Zimmerman et al. ${ }^{20}$, em que docentes afirmaram dificuldade em avaliar e formular provas por falta de embasamento teórico, além da ausência de padronização no curso de Medicina.

A importância da avaliação de desempenho, nesse nível de formação, deve-se à sua potencialidade para verificar habilidades clínicas (comunicação, exame físico e procedimentos), e a avaliação contínua e formativa permite a correção de falhas e reduz a possibilidade de erros.

Ross et al. ${ }^{21}$, em um estudo de coorte retrospectivo, analisaram o desempenho e a progressão dos médicos residentes quando avaliados por um sistema tradicional de avaliação somativa, comparado a um sistema de avaliação baseado em competências. Nessa perspectiva, evidenciaram a eficácia da abordagem dos residentes com dificuldades, com foco na possível correção das falhas. Os métodos avaliativos tradicionais identificavam os problemas dos residentes, mas não eram eficazes na correção das lacunas apresentadas, talvez porque as avaliações fossem desconectadas das observações diárias.

Nesta pesquisa, observou-se que o método mais utilizado é a avaliação global, com 76,2\% (16/21) das respostas, seguida da observação do aluno em ambiente real, utilizada por 43\% (9/21) dos participantes. Quanto à avaliação em ambiente simulado, dois participantes $(9,5 \%)$ relataram alguma formação para esse modelo, embora não o utilizem com os residentes. Os outros instrumentos citados referiam-se a métodos avaliativos cognitivos, como discussão de casos clínicos (10/21 ou $47,6 \%)$, testes de múltipla escolha (7/21 ou 33,3\%) e avaliação oral (7/21 ou 33,3\%) (Gráfico 1).

A avaliação global consiste em uma escala que avalia conhecimento, pontualidade e atitude. Segundo dados da literatura que corroboram os achados deste artigo, a avaliação global é o instrumento mais utilizado por todos os cursos de pós-graduação para avaliação de competências nos Estados Unidos $^{22}$, por exemplo. Contudo, os autores observaram duas grandes limitações da avaliação global. A mais importante delas diz respeito ao fato de que um médico com deficiência em uma área poderá atingir uma classificação global satisfatória se demonstrar destaque em outra competência. A outra limitação é que esse instrumento fornece pouca ou nenhuma informação para um feedback construtivo, um componente importante do desenvolvimento dos médicos residentes ${ }^{23}$.

Para validar o conceito global, a proposta é a construção de uma matriz com itens específicos, que reflitam a combinação de atributos necessários ao bom desempenho profissional, além de o residente ser avaliado por diversos docentes ${ }^{23}$.

A observação do aluno em ambiente real é um método usual de avaliação, de fácil operacionalização, propício à provisão de feedback imediato e com boa aceitação dos participantes². No entanto, a ausência de sistematização, a não

Gráfico 1. Distribuição dos métodos avaliativos utilizados pelos docentes e preceptores da residência médica em pediatria do Hospital Universitário da Ufal, 2018.

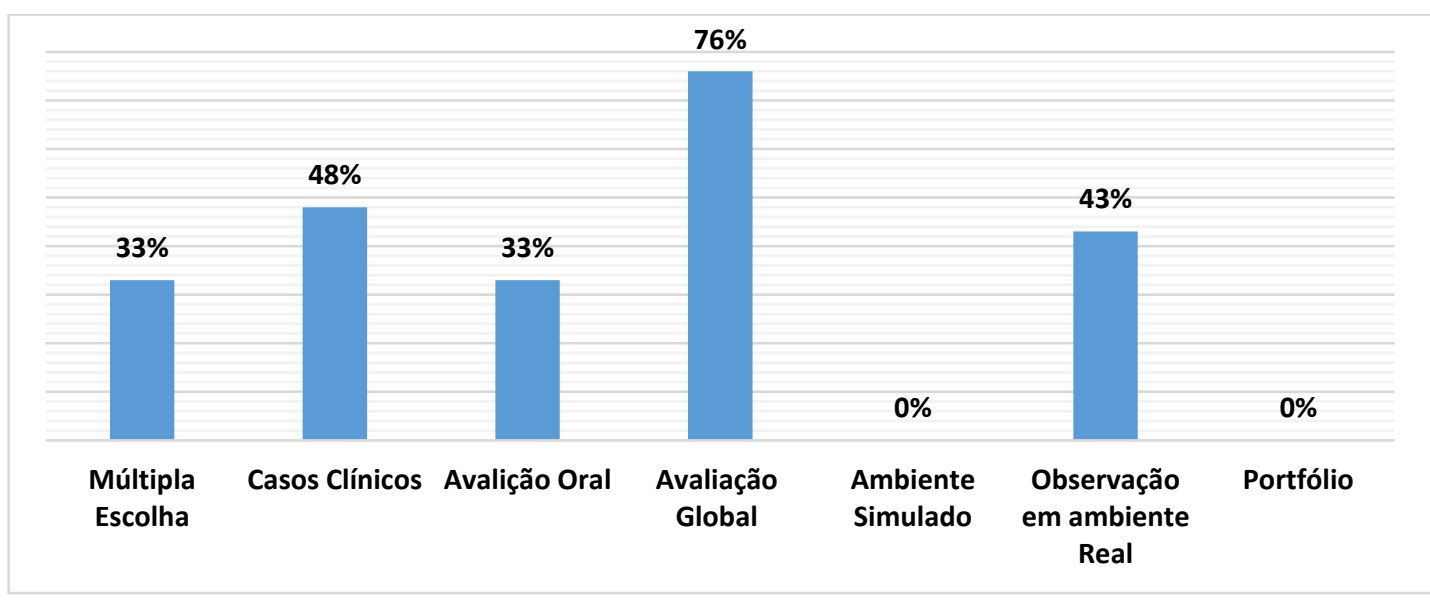


utilização de formulário de avaliação (checklist) ou a falta de feedback gera um baixo grau de fidedignidade.

A avaliação em ambiente simulado permite abordar tarefas clínicas que representam o que é comum na prática médica, em nível de dificuldade compatível com o estágio da formação, e assegurar que todos os alunos sejam avaliados em condições semelhantes, com observação cuidadosa e com a utilização de checklist $^{2}$. No entanto, nenhum dos participantes utiliza esse método com os residentes, apesar de dois deles o conhecerem. Esse fato pode sugerir a ausência de um processo institucionalizado de avaliação, a falta de interação entre os participantes e a própria dificuldade para sua operacionalização, por ser um método que demanda um trabalho em equipe, uma execução prolongada e uma logística mais onerosa (cenários, atores, formulários de avaliação).

Utilizar métodos avaliativos de habilidades clínicas, quer em ambientes reais ou simulados, não é uma tarefa fácil, pois envolve a escolha do método mais apropriado e que recaia em uma técnica válida, fidedigna, viável e aceitável por todos os envolvidos no processo ${ }^{2}$. Implica, também, a formação docente em avaliação, o trabalho em equipe, a participação em pesquisas-ação e outras estratégias, além de padronização e sistematização. Alguns participantes manifestaram-se positivamente em relação à pesquisa e à necessidade de padronização da avaliação:

Que a partir dessa pesquisa e dos resultados obtidos, haja uma padronização dos métodos avaliativos utilizados na Faculdade de Medicina. É muito importante padronizar as avaliações (D1).

Ser projeto, cadastrado na gerência de ensino, incluindo preceptores de todas as clínicas (D4).

Nesse sentido, 90\% dos participantes referiram a necessidade de treinamento em métodos avaliativos e demonstraram interesse em participar de uma capacitação sobre um método avaliativo específico em ambiente simulado, como explicitado na fala de P2: "Tenho interesse em capacitações para a docência". Ou, ainda, quando se aborda a necessidade de um método avaliativo de desempenho como o OSCE: "Inserir o OSCE como avaliação integrada no internato de pediatria no $9^{\circ}$ e $10^{\circ}$ períodos (ao menos $1 \mathrm{x} /$ semestre)" (D5).

O aprimoramento do docente e preceptor em avaliação assegura a qualidade do avaliar e do processo ensinoaprendizagem, uma vez que a avaliação permite a revisão do planejamento educacional e ajustes na sua prática docente ${ }^{20}$.

Um dos depoimentos remete à necessidade de feedback comentada por D3: "Avaliação contínua, no cotidiano, é fundamental para o processo de aprendizagem, sempre com feedback para o seu fortalecimento". Observa-se, nesse depoimento, que o docente sintetiza todo o processo de uma avaliação com função formativa.

O feedback é o substrato de uma avaliação formativa e uma ferramenta efetiva para melhorar o desempenho do estudante, principalmente quando é feito de imediato, após a tarefa clínica ${ }^{10}$. Para tanto, deve se dar de forma dialógica, com o aluno executando um papel importante na avaliação do seu próprio desempenho 24 .

Na educação médica, o feedback é essencial tanto para os educadores, de modo que possam promover aprendizagens de qualidade, quanto para os alunos. O feedback fornece informações sobre a qualidade do trabalho realizado, com o propósito de gerar melhorias ${ }^{25}$.

Os resultados mostraram que a preocupação dos participantes comos métodos avaliativos dos residentes era coletiva, considerando ser esse o primeiro passo para a institucionalização de mudanças dentro do cenário de ensino-aprendizagem.

\section{Etapa 2 - Workshop: dinâmica integradora e interativa}

Após a identificação do problema, evidenciado pela ausência de sistematização, institucionalização e formação docente quanto a métodos avaliativos para o residente em pediatria, uma intervenção foi planejada e executada como estratégia de desenvolvimento docente em avaliação. A maioria dos participantes optou no treinamento pelo OSCE.

O OSCE é um instrumento de avaliação em ambiente simulado, situado na dimensão "mostrar como faz" da pirâmide de Miller. É um dos métodos avaliativos mais utilizados e apreciados em várias partes do mundo. Quando bem elaborado, fornece informações importantes para a atuação do futuro profissional.

No workshop com atividade simulada, compareceram nove (43\%) participantes da pesquisa: sete docentes e dois preceptores, pouco menos de $50 \%$ da amostra total, apesar dos horários previamente consensuados. Compareceram, ainda, observadores e convidados, como gestores da residência em pediatria, médicos residentes e monitores da disciplina de pediatria. A atividade foi realizada na Faculdade de Medicina da Ufal, localizada ao lado do Hupaa. Segundo descrito por Steinert ${ }^{4}$, a proximidade do local de trabalho é um dos fatores facilitadores para aumentar a participação, a motivação e o acesso desses educadores. Dos presentes, oito eram docentes e preceptores vinculados ao Hupaa. Observou-se que apenas um dos seis participantes vinculados às outras instituições compareceu. Justificativas sobre a ausência foram comunicadas.

O workshop consistiu em dois momentos sequenciais: no primeiro, os pesquisadores expuseram dados gerais sobre avaliação e sobre o método OSCE (em sala de aula); a segunda 
parte foi direcionada à prática do workshop, realizado, nas salas de tutoria da Faculdade de Medicina, com os atores e manequins do laboratório de habilidades.

Nesse momento, os participantes da pesquisa foram orientados a rodiziar pelos vários cenários, em grupos de três ou quatro. Enfatize-se que o deslocamento nas estações, no OSCE, é feito pelo estudante avaliado, porém, nessa ocasião, o objetivo não foi avaliar o residente, mas possibilitar que o docente e o preceptor participantes pudessem vivenciar uma experiência com diferentes cenários, formulários de avaliação e feedbacks.

Ao final do circuito, os participantes retornaram à sala de aula, onde foram apresentados os passos do OSCE realizado, com os registros fotográficos do workshop.

\section{Etapa 3 - Avaliação do impacto do workshop}

Nessa etapa, para avaliar o desdobramento do workshop sobre o OSCE, empregou-se o método de Kirkpatrick. Nesse sentido, o nível 1 do método de Kirkpatrick (reação), utilizado na avaliação do treinamento, dimensiona as impressões dos participantes em relação ao conteúdo, aos instrutores, aos materiais e recursos, ao ambiente e às instalações. De acordo com Kirkpatrick, todos os programas devem ser avaliados, nesse nível, como forma de promover melhorias. A reação positiva não garante, necessariamente, a aprendizagem, mas a reação negativa e a insatisfação, com certeza, reduzem as possibilidades de aprendizagem ${ }^{26,27}$. Nesse aspecto, os 12 itens da avaliação obtiveram predomínio de avaliações positivas, sendo dez itens avaliados entre excelente e bom.

No item relativo à aplicação do método avaliativo em treinamento (OSCE), todos os participantes mostraram-se favoráveis a utilizá-lo em sua prática de ensino-aprendizagem com os médicos residentese, também, com os estudantes da graduação. Isso corrobora os comentários sobre a motivação para frequentar o workshop, quando se referem, basicamente, ao desenvolvimento docente em métodos avaliativos mais inovadores:

Novas aprendizagens (D1).

Avaliação dos discentes (D3).

Aprimorar o processo de avaliação (D6).

Melhorar minha atuação com os residentes $e$ estudantes (P1).

$\mathrm{Na}$ atividade, oportunizou-se, também, a participação de mestrandos, do mestrado profissional em ensino na saúde, da disciplina de avaliação do ensino, como uma forma de aprendizagem por meio da observação da execução do método em tempo real.

Reforça-se que uma ação positiva em equipe sofre uma idealização e gera uma nova ação, conforme os ciclos da pesquisa-ação educacional. Destaca-se, ainda, que o treinamento atingiu o nível 3 de Kirkpatrick, visto que os participantes utilizaram o método avaliativo do aprendizado na sua prática cotidiana, agora com os internos. A atividade ocorreu na mesma estrutura do workshop realizado, o que corrobora o pensamento de Steinert ${ }^{4}$, quando diz que levar as atividades de desenvolvimento docente para o local de trabalho aumenta a participação, a motivação e o acesso, e, com o engajamento individual, um sequenciamento de atividades e orientação continuada dos participantes assegura uma melhor eficácia do treinamento.

O efeito borboleta ${ }^{28}$ causado pela intervenção (workshop), na proposição da pesquisa-ação educacional, e pelo método avaliativo em si transformou-se, também, em um ambiente de ensino-aprendizagem para alunos de outros níveis de formação. Espera-se que esse efeito, metáfora usada na ciência, descrito por Edward Lorenz em $1972^{28}$, causado por uma pequena mudança no início de um evento, possa trazer consequências futuras positivas e que se desdobre em novas intervenções em métodos avaliativos na instituição, para todos os níveis de graduação e pós-graduação. Nesse sentido, é na educação e nas reflexões daí advindas que se dá o potencial transformador de mudanças.

As limitações do estudo apontam para a dificuldade em agregar todos os participantes envolvidos (docentes, preceptores e atores), por conta da conciliação de horário, local e trabalho. Além disso, em razão da logística e praticidade da organização, o estudo abordou apenas um método de avaliação.

\section{CONSIDERAÇÕES FINAIS}

A pesquisa-ação realizada com os docentes e preceptores que atuam na residência médica propiciou, principalmente, identificar limitações no sistema de avaliação e feedback do médico residente em pediatria, como a inadequação dos métodos utilizados e a percepção de que os docentes e preceptores devem estar mais alinhados com a avaliação formativa e o feedback. Nesse sentido, deve abranger todos os elementos do ensino-aprendizagem na prática em serviço, tais como habilidades clínicas, atitude, ética, raciocíno clínico e profissionalismo, permitindo assim a identificação e correção de eventuais falhas do médico em formação.

A metodologia utilizada na pesquisa revelou um efeito agregador e contribuiu para desenvolver o sentido colaborativo e integrativo no grupo, despertando, por meio do protagonismo, a necessidade de formação docente. A partir daí, algumas mudanças positivas foram observadas nos participantes (professores e preceptores) com relação à avaliação em outros níveis do ensino-aprendizagem (graduação e internato). 
Conquanto tenham sido geradas reflexões sobre a inadequação do processo avaliativo, a intervenção (workshop) não foi suficiente para interferir positivamente, em curto prazo, na avaliação da residência médica em pediatria.

Sabe-se que um processo avaliativo bem elaborado exige tempo, periodicidade, planejamento e organização. Mudanças sólidas requerem outros fatores além de formação docente continuada no ensino-aprendizagem, exigem dedicação, comprometimento dos educadores, docentes e preceptores envolvidos, conscientização do discente e uma formalização institucional de fato.

Avaliar é um processo complexo e permanece um desafio, no entanto promover espaços para debates e reflexões, como demonstrou a pesquisa, pode levar a medidas significativas e evoluir para os objetivos educacionais almejados para o médico em formação.

\section{CONTRIBUIÇÃO DAS AUTORAS}

Bruna de Sá Duarte Auto participou da coleta, análise, interpretação e discussão dos dados, e da redação e revisão do texto. Maria Viviane Lisboa de Vasconcelos orientou a pesquisa e participou da análise, interpretação e discussão dos dados, e da redação e revisão do texto. Ana Lydia Vasco de Albuquerque Peixoto participou da discussão dos dados e da revisão do texto.

\section{CONFLITO DE INTERESSES}

Declaramos não haver conflito de interesses.

\section{FINANCIAMENTO}

Declaramos não haver financiamento.

\section{REFERÊNCIAS}

1. Megale L, Gontijo ED, Motta JAC. Avaliação de competência clínica em estudantes de medicina pelo Miniexercício Clínico Avaliativo (Miniex). Rev Bras Educ Med. 2009;33(2):166-75.

2. Troncon LEA. Avaliação de habilidades clínicas: os métodos tradicionais e o modelo "OSCE". Olho Mágico. 2001;1:8-12.

3. Perim GL, Abdalla IG, Aguilar-da-Silva RH, Lampert JB, Stella RCR, Costa NMSC. Desenvolvimento docente e formação médica. Rev Bras Educ Med. 2009;33(1):70-82.

4. Steinert Y. Faculty development: from rubies to oak. Med Teach. 2019;41, p. 429 - 435. doi: 10.1080/0142159X.2019.1688769.

5. Hospital Universitário Professor Alberto Antunes. Comissão de Residência Médica. Relatório. Maceió: Hupaa; abr. 2020.

6. Brasil. Resolução CNRM nº5 de 12 de novembro de 1979. Estabelece especialidades médicas credenciáveis como Programa de Residência Médica e dá providências adicionais. Brasília: Ministério da Saúde, Comissão Nacional de Residência Médica; 1979.
7. Brasil. Resolução CNRM n 02, de 17 de maio de 2006. Dispõe sobre requisitos mínimos dos Programas de Residência Médica e dá outras providências. Brasília: Ministério da Saúde, Comissão Nacional de Residência Médica; 2006 [access in 23 jun 2019]. Available from: http:// portal.mec.gov.br/index.php?option=com_docman\&view=download\& alias=100951-resolucao-cnrm-n-2-de-17-de-maio-de-2006\&category_ slug=novembro-2018-pdf\&ltemid=30192.

8. Souza RGS. Atributos fundamentais dos procedimentos de avaliação. In Tibério IFLC, Daud-Gallotti RM, Troncon LEA, Martins MA, organizadores. Avaliação prática de habilidades clínicas em medicina. São Paulo: Atheneu; 2012. p. 1-11.

9. Panúncio-Pinto MP, Troncon LEA. Avaliação do estudante: aspectos gerais. Medicina (Ribeirão Preto). 2014;47(3):314-23.

10. Zeferino AMB, Passeri SMRR. Avaliação da aprendizagem do estudante. Cadernos ABEM. 2007;3:39-43.

11. Zaidan S, Ferreira MCC, Kawasaki TF. A pesquisa da própria prática no mestrado profissional. Revista Plurais. 2018;3(1):88-103.

12. Thiollent M. Metodologia da pesquisa-ação. São Paulo: Cortez; 2002.

13. Tripp D. Pesquisa-ação: uma introdução metodológica. Educação e Pesquisa. 2005;31(3):443-66.

14. Malheiros BT. Metodologia da pesquisa em educação. Rio de Janeiro: LTC; 2011.

15. Bardin L. L'analyse de contenu. 2ème ed. Paris: Presses Universitaires France; 2013.

16. Kirkpatrick J, Kirkpatrick WK. The Kirkpatrick four levels: a fresh look 50 years 1959-2009. Kirkpatrick Partners; 2009 [access in $1^{\circ}$ nov 2019]. Available from: http://www.kirkpatrickpartners.com/Portals/0/Resources/ Kirkpatrick\%20Four\%20Levels\%20white\%20paper.pdf.

17. Botti SHO. O papel do preceptor na formação de médicos residentes um estudo de residências em especialidades clínicas de um hospital de ensino [tese]. Rio de Janeiro: Escola Nacional de Saúde Pública Sergio Arouca; 2009

18. Almeida MTC, Batista NA. Ser docente em métodos ativos de ensino-aprendizagem na formação do médico. Rev Bras Educ Med. 2011;35(4):468-76.

19. Norcini J, Anderson MB, Bollela V, Burch V, Costa MJ, Duvivier R, et al. Consensus framework for good assessment. Med Teach. 2018, 40(11):1102-9.

20. Zimmermann MH, Silveira RMCF, Gomes RZ. O professor e a arte de avaliar no ensino médico de uma universidade no Brasil. Rev Bras Educ Med. 2019;43(3), p. 5-15.

21. Ross S, Binczyk NM, Hamza DM, Schipper S, Humphries P, Nichols D, et al. Association of a competency-based assessment system with identification of and support for medical residents in difficulty. JAMA Netw Open. 2018;1(7):1-12 [access in 30 nov 2019]. Available from: https:// jamanetwork.com/journals/jamanetworkopen/fullarticle/2713042.

22. Silber GC, Nasca TJ, Paskin DL, Eiger G, Robeson M, Veloski JJ. Do global rating forms enable program directors to assess the ACGME competencies? Acad Med. 2004;79(6):549-56.

23. Domingues $\mathrm{RCL}$, Amaral E, Bicudo-Zeferino AM. Conceito global: um método de avaliação de competência clínica. Rev Bras Educ Med. 2009;33(1):148-51.

24. Ramani S, Krackov SK. Twelve tips for giving feedback effectively in the clinical environment. Med Teach. 2012;34:787-91.

25. Qureshi NS. Giving effective feedback in medical education. Obstet Gynaeco. 2017;19:243-8. doi: 10.1111/tog.12391.

26. Mira VL, Peduzzi M, Melleiro MM, Tronchin DMR, Prado MFF, Santos PT, et al. Análise do processo de avaliação da aprendizagem de ações educativas de profissionais de enfermagem. Rev Esc Enferm USP. 2011;45:1574-81 [access in 19 sep 2019]. Available from: http://www.scielo.br/scielo.php?script=sci arttext\&pid=S0080-62342011000700006\&lng=en\&nrm=iso.

27. Silva NP, Francisco AC, Hatakeyama K, Silva MCG. Avaliando as práticas de educação corporativa à distância por meio do modelo de Kirkpatrick: um estudo de caso numa empresa do ramo de energia no estado do Paraná Emancipação. 2010;10(2):501-15.

28. Ferrari PC, Angotti JAP, Tragtenberg MHR. Educação problematizadora a distância para a inserção de temas contemporâneos na formação docente: uma introdução à Teoria do Caos. Ciênc Educ (Bauru). 2009;15(1):85-104. 